\title{
Purtscher's Retinopathy Associated with Tortuous Internal Carotid Artery
}

\author{
Soo Park, ${ }^{1}$ Lik Thai Lim, ${ }^{1}$ Timothy Fetherston ${ }^{2}$ \\ ${ }^{1}$ Tennent Institute of Ophthalmology, Gartnavel General Hospital, Glasgow, and ${ }^{2}$ Department \\ of Ophthalmology, Sunderland Eye Infirmary, City Hospitals Sunderland NHS Trust, \\ Sunderland, UK
}

This report is of a 71-year-old woman with unilateral non-traumatic left Purtscher's retinopathy following shoulder exercises. She developed blurred vision in the left eye a few hours after the exercises. The only clinical findings of note were an ipsilateral tortuous internal carotid artery and multiple cotton wool spots around the left macula region consistent with Purtscher's retinopathy. To the authors' knowledge, this is the first report illustrating internal carotid artery tortuosity as an underlying rare cause of Purtscher's retinopathy.

Key words: Carotid artery, internal, Retinal diseases

Asian J Ophthalmol. 2011;12:179-80.

\section{Introduction}

Purtscher's retinopathy was first described in 1910 in patients who sustained severe head trauma. Similar retinal lesions characterised by bilateral multiple areas of retinal haemorrhages and whitening have been subsequently observed in other traumatic conditions (long bone fracture and chest compression injuries) and non-traumarelated conditions (acute pancreatitis, chronic renal failure, and connective tissue disorders). ${ }^{1}$ Patients typically present with acute painless bilateral loss of vision within 24 to 48 hours of the onset of illness. The degree of visual loss may vary, with most patients having visual acuities of $6 / 60$ or worse. ${ }^{2}$ Acute retinal features usually consist of cotton wool spots followed by retinal haemorrhages, with Purtscher flecken being the least prevalent. Patients with an absence of retinal haemorrhages have been reported. ${ }^{2}$

\section{Case Report}

A 71-year-old woman presented in 2008 with sudden onset of blurring of vision in the left eye within a few hours of doing shoulder and neck exercises. She had cervical spondylosis and was receiving physiotherapy, which involved gentle stretching exercises of her shoulders and neck. Her past medical history included systemic hypertension, for which she was taking treatment.

Correspondence: Dr Lik Thai Lim, Tennent Institute of Ophthalmology, Gartnavel General Hospital, 1053 Great Western Road, Glasgow G12 OYN, UK.

Tel: (44 78) 43617788 ;

E-mail: likthai@doctors.org.uk
At presentation, her best-corrected visual acuity was $6 / 9$ in the right eye and $6 / 18$ in the left eye. Fundal examination showed scattered cotton wool spots at the posterior pole of the left eye (Figure 1). The right fundus was unremarkable. Humphrey visual field tests were of limited reliability, but appeared to show a generalised constriction on the left side. Haematological and biochemical tests were unremarkable and did not reveal any relevant underlying systemic problem. She was referred to the cardiologist, and echocardiogram and carotid Doppler studies were performed. These investigations were unremarkable and showed a normal appearance of the aortic valves and no significant carotid stenosis, respectively. The only abnormality that

Figure 1. Left fundus photograph showing scattered cotton wool spots.

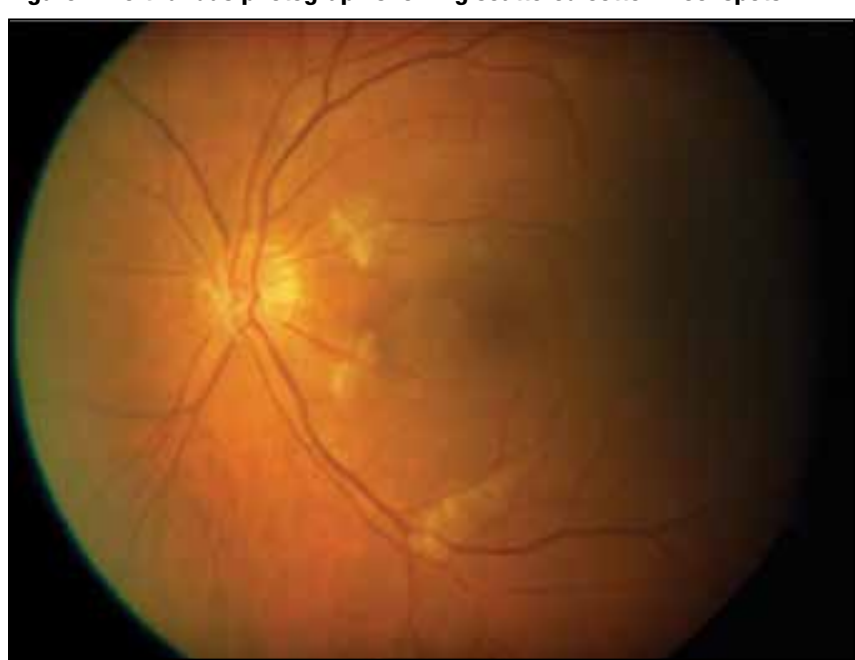


was detected was a markedly tortuous left internal carotid artery (ICA).

The history clearly pointed towards a precipitating event, with subsequent delay of the onset of visual symptoms. A preliminary diagnosis of unilateral Purtscher's retinopathy was made. A differential diagnosis of grade 3 hypertensive retinopathy was considered, but was thought to be highly unlikely due to the presence of acute blurring of vision.

She was treated conservatively, with observation only. After 3 months, her vision had markedly improved to $6 / 6$ in the left eye.

\section{Discussion}

It is postulated that the underlying mechanism of Purtscher's retinopathy is occlusion of small pre-capillary retinal arterioles by embolisation of intermediate-sized microparticles, consisting of fat, air, leucocytes, platelets, fibrin, or products of complement activation. ${ }^{2.3}$ Despite reported cases of unilateral involvement, this is an uncommon finding, with most individuals being bilaterally affected. ${ }^{4}$ Importantly, there have been no previous reports of a carotid artery abnormality, in particular a tortuous ICA, as an underlying cause of Purtscher's retinopathy.

Studies have shown an association between tortuous ICA with cerebrovascular insufficiency. ${ }^{5}$ It is understood that the thromboembolic mechanism is a result of turbulence of blood flow at the site of arterial tortuosity. This causes endothelial damage and generation of embolic particles, which may lead to a cerebrovascular event. ${ }^{5}$ For this patient, other underlying traumatic or systemic related conditions accounting for Purtscher's retinopathy were excluded, and the only abnormality detected was a tortuous left ICA. Based on the anatomy of the ICA, which begins at the bifurcation of the common internal carotid artery (level of C4), it was speculated that the neck and shoulder exercises could have released embolic particles originating from within the tortuous ICA that were sufficiently small to have occluded the pre-capillary retinal arterioles.

This patient demonstrates that ophthalmologists should be aware that patients presenting with non-traumatic Purtscher's retinopathy who have no underlying systemic medical conditions should be examined for carotid artery abnormalities and managed appropriately through a multidisciplinary approach.

\section{References}

1. Gass JDM. Stereoscopic atlas of macular diseases: diagnosis and treatment. 2nd ed. St Louis: Mosby; 1987.

2. Agrawal A, McKibbin M. Purtscher's retinopathy: epidemiology, clinical features and outcome. Br J Ophthalmol. 2007;91: 1456-9.

3. Behrens-Baumann W, Scheurer G, Schroer H. Pathogenesis of Purtscher's retinopathy. An experimental study. Graefes Arch Clin Exp Ophthalmol. 1992;230:286-91.

4. Burton T. Unilateral Purtscher's retinopathy. Ophthalmology. 1980;87:1096-105.

5. Ballotta E, Abbruzzese E, Thiene G, et al. The elongation of the internal carotid artery: early and long-term results of patients having surgery compared with unoperated controls. Ann Vasc Surg. 1997;11:120-8. 